\title{
AN ANTHOLOGY OF THE DISTINGUISHED ACHIEVEMENTS IN SCIENCE AND TECHNIQUE. PART 34: DISCOVERY AND STUDY OF QUANTUM-WAVE NATURE OF MICROSCOPIC WORLD OF MATTER
}

Purpose. Implementation of brief analytical review of the basic distinguished scientific achievements of the world scientistsphysicists in area of discovery and study of quantum-wave nature of physical processes and phenomena flowing in the microscopic world of circumferential people matter. Methodology. Scientific methods of collection, analysis and analytical treatment of scientific and technical information in area of theoretical and experimental physics, devoted the results of researches of quantum and physical processes flowing in nature on atomic and subatomic levels. Results. The brief scientific and technical review of the basic scientific discovery and achievements of scientists-physicists is resulted in area of structure of atom of matter, generation, radiation, distribution and absorption of physical bodies of short-wave hertzian waves, indicative on a dominating role in the microscopic financial world of positions and conformities to the law of wave (by quantum) mechanics, carrying especially probabilistic character a microstructure. Originality. Systematization is executed with exposition in the short concentrated form of the known materials on the quantum theory (electromagnetic) of caloradiance, quantum theory of atom, electronic waves, quantum theory of actinoelectricity, quantum statistics of microparticless, quantum theory of the phenomenon superfluidity of liquid helium, quantum electronics and quantum-wave nature of drift of lone electrons in the metal of explorers with an electric current. Practical value. Popularization and deepening of fundamental physical and technical knowledges for students and engineer and technical specialists in area of classic and quantum physics, extending their scientific range of interests, and also support a further scientific study by them surrounding nature and to development of scientific and technical progress in society. References 27 , figures 15.

Key words: quantum theory of thermal radiation, quantum theory of atom, electron waves, quantum theory of photoelectric effect, quantum statistics of microparticles, quantum theory of the phenomena of superfluidity of helium, quantum electronics, quantum wave nature of the drift of electrons in metal, review.

Приведен краткий аналитический обзор основных научных достижсенй ученых в области открытия и изучения квантово-волновой природы физических процессов и явлений, протекающих в микромире окружающей нас материи. В число таких достижений вочли как ставщие классическими квантовые теории теплового излучения абсолютно черного тела, атома, фотоэлектрического эффекта и явления сверхтекучести жсикого гелия, так и современные открытия в области квантовой электроники и новейшие квантовомеханические результаты для дрейфа свободных электронов и их волновых распределений в проводниках электрических цепей с током. Библ. 27, рис. 15.

Ключевые слова: квантовая теория теплового излучения, квантовая теория атома, электронные волны, квантовая теория фотоэффекта, квантовая статистика микрочастиц, квантовая теория явления сверхтекучести гелия, квантовая электроника, квантово-волновая природа дрейфа электронов в металле, обзор.

Introduction. In the process of cognition of the surrounding nature people accumulate a certain «baggage» (margin) of scientific and technical knowledge, we have understood that humanity is interesting and important for their life living on Earth, and the constant time-development of industrial production and a variety of advanced technologies not only the macrocosm but microcosm defining occurrence in nature the various physico-chemical and biological processes and the macroscopic properties of matter. It is necessary to remind the reader that, according modern ideas about the structure of matter at the microcosm is understood as set placed in a vacuum of material objects (bodies) with linear dimensions of $10 \mathrm{~nm}$ or less [1]. Therefore, practically since the end of the 19th century, physicists and chemists in the extent of its theoretical possibilities and means at their disposal physical and chemical tools have been actively engaged in the study of microscopic properties of matter and at the same time opening new physical and chemical phenomena. The first in a series of similar discoveries was the discovery of outstanding
German experimental physicist Wilhelm Roentgen (1845$1923)$ in 1895 receiving in the near future the name « $X$ rays» and marked one of the first Nobel Prizes in physics for 1901 [2, 3]. Then, in 1896 the eminent French physicist Henri Becquerel (1852-1908) discovered the phenomenon of «radioactivity» named in 1898 to study its outstanding French physicists and chemists Marie Sklodowska-Curie (1867-1934) and Pierre Curie (18591906) and for which in 1903 Becquerel and $M$. Sklodowska-Curie and Pierre Curie who discovered at that time, such as a radioactive chemical elements polonium $P o$ and radium $R a$ were awarded the Nobel Prize in physics [2, 3]. We mention the fact that for the release of radioactive radium $R a$ in pure metallic form, and a comprehensive study of it as a chemical element in the periodic system of elements of D.I. Mendeleev [4] M. Sklodowska-Curie was awarded the $[2,3]$ for the second time the Nobel Prize in chemistry for 1911. Today such an honor is not honored more than any woman in the world. In 1935, the eldest daughter of M. Sklodowska- 
Curie and Pierre Curie Irène Curie (1897-1956) and her husband Frederic Joliot-Curie (1900-1958) became prominent French scientists-physicists, for the discovery of a fundamental phenomenon of «artificial radioactivity» won the Nobel Prize in chemistry [2, 3]. Returning again to the end of the 19th century, we note that in 1897 , an outstanding British physicist Joseph John Thomson (1856-1940) elementary carriers of electricity (a kind of «quanta of electricity») were open - electrons [2, 3]. During this scientific discovery J.J. Thomson was awarded the Nobel Prize in physics for 1906 [3, 5]. The quantitative value of the negative charge of an electron $e_{0}=1.602 \cdot 10^{-19} \mathrm{~K}$ is a global constant has been determined empirically with a surprisingly high degree of accuracy (with measurement errors of up to 1\%) in 1917 by outstanding American experimental physicist Robert Millikan (1868-1953) [2]. The results of these experimental studies R. Millikan was highly appreciated by the international scientific community and awarded the Nobel Prize in physics for 1923 [3, 5].

To the above it should be added that in fact even in 1886 by the German physicist Eugen Goldstein (18501930) who first introduced to electrophysics in 1876 the term "cathode rays» in a two-electrode discharge tube filled with the most abundant naturally occurring isotope of hydrogen protium ${ }_{1}^{1} \mathrm{H}$ were opened «canal rays» and the elementary particles such as protons are present in them having a positive electric charge $[3,6]$. At that time the values of their charge or their weight are not yet known. It is much later experimental physicists significantly, it was found that the modulus of the charge is equal to the module of the electron charge $e_{0}$ and their mass $m_{p}$ about 1836 times more the mass of the rest electron $m_{e}[1,4]$. In physics, it is considered, however, that the proton as an elementary particle, «embossed» by alpha-particle of the atomic nucleus opened in the period 1914-1919 by a group of talented British atomic physicists including E. Rutherford, E. Marsden and J. Nuttall $[3,4]$. It is necessary to specify the fact that this is extremely important for the understanding of the structure of a microcosm of the earth and nature of our universe as a whole electrically neutral elementary particle, the neutron was discovered in the course of experimental atomic research with the help of alpha particles in 1932 by a member of the famous school of physics of Ernest Rutherford outstanding British experimental physicist James Chadwick (1891-1974) [3, 5]. The mass of the neutron $m_{n}$ with zero total electric charge in accordance with the experimental data of modern nuclear measurements was found to be about $1839 m_{e}[1,4]$.

In the world history of the development of physics teaching on the atom of matter and the structure of the theory is of a special place. The reason for that is the fundamental nature of these issues, the underlying structure of matter. By 1913, thanks to the results of experimental research using the $\langle X$-ray» a talented young British physicist Henry Moseley (1887-1915) who was tragically killed on the battlefields of World War I was installed one of the main physical characteristics of the substance of the atom - the value of positive charge its nucleus, located in the center of this microformations [2, 5]. This value is later called «the number of Mendeleev»» corresponds to the sequence number $Z$ of the chemical element in the periodic system of the fundamental elements of the D.I. Mendeleev [4]. Using the experimental results of his student an outstanding British physicist Ernest Rutherford (1871-1937) in 1913, after years of deliberation and searching proposed to scientific world known planetary-nuclear model of the atom [3, 4]. It should be noted that the precursor of such a momentous event in the physico-chemical studies of world steel Rutherford and his other young talented student, the British physicist and chemist Frederick Soddy (18771956) who became the winner of the Nobel Prize in chemistry for 1921 [3], in the theory and practice of the radioactive decay of chemical elements. For scientific achievements in describing patterns of radioactive transformation of atoms like without external interference, and bombarded emanating from the radioactive source containing a capsule of radium $R a$, by alpha particles - double ionized helium atoms ${ }_{2}^{4} \mathrm{He}$ and in fact by their nuclei, E. Rutherford was awarded the Nobel Prize in chemistry for 1908 [3].

\section{Max Planck - founder of the quantum theory} of thermal radiation. At the turn of the 19th and 20th centuries in physical science, new concepts and theories, significantly changed as a scientific «front», and internal scientific «stuffing» the seemingly immutable «building» of classical physics. In 1897 the talented German physicist Max Planck (Fig. 1) started the theoretical study of the dynamics of thermal radiation of the absolute blackbody (ABB). It is necessary to remind the reader that ABB considered a physical body that absorbs all incident on the surface of the thermal (electromagnetic) radiation, regardless of wavelength and the body temperature [4]. Of course, that ABB is an idealized physical body, absent in nature, but is often used by us as a calculated thermophysical model.

In 1900 Max Planck based on a fundamentally new and revolutionary approach in fact showed that the emission or absorption of the physical body of the thermal radiation, which has, by the way, an electromagnetic nature $[4,7]$, is not continuous, as anticipated earlier in classical physics and intermittently. And, and, these processes occur individual energy portions, which he called «quanta of action» [3, 4]. The value of the photon energy $E_{n}$ is a multiplication of the fundamental constant $h=6.626 \cdot 10^{-34} \mathrm{~J} \cdot \mathrm{s}$ later called in physics as the Planck constant [3] on the quantized frequency $v_{n}(n=1,2,3, \ldots$ integer quantum number) of radiation waves (absorption) $[4,7]$. For the discovery of discrete quanta of energy (action) M. Planck was awarded Nobel Prize in Physics for $1918[3,6]$. 


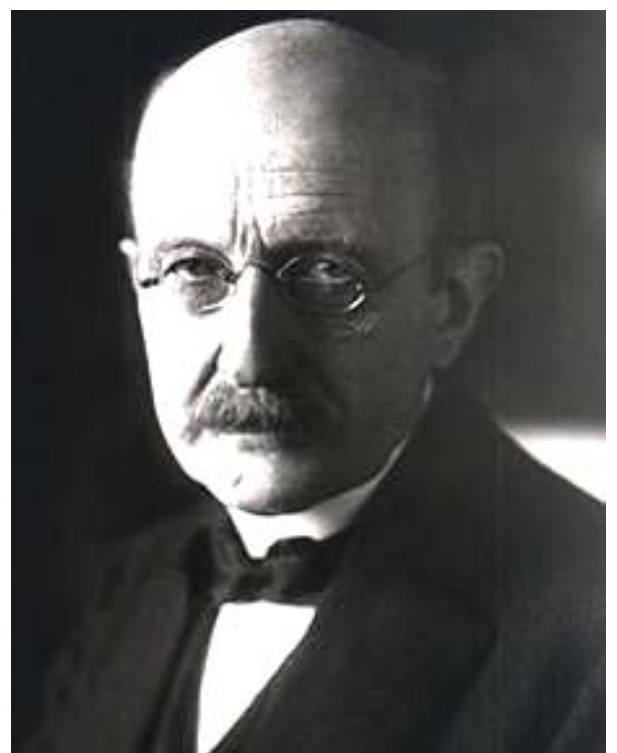

Fig. 1. Prominent German theoretical physicist Max Planck (1858-1947)

2. Niels Bohr and the creation of a quantumnuclear model of the atom. For all its physical and progressive values proposed by E. Rutherford within the framework of classical mechanics and electrodynamics planetary nuclear model of the atom, containing in the central part of the positively charged nucleus and around the periphery of a negatively charged electron shell of a sphere-like, had a significant and insurmountable flaw. This theoretical model is electrically neutral atom with rotating around a fixed core bound electrons was unstable electrodynamic system. According to the laws of classical electrodynamics moving electrons in such a model were to continuously radiate electromagnetic energy and eventually «fall» into the nucleus $[4,6]$. In nature these subatomic processes are observed. Using quantum theory of the outstanding German theoretical physicist Max Planck, the eminent Danish physicist Niels Bohr (18851962) in 1913 to overcome the above-mentioned fundamental lack of planetary nuclear model of the atom by Rutherford proposed a new hypothesis (in Greek the word «hypothesis» refers to namely «scientific hypothesis» [8]) that the atom of matter absorbs and emits electromagnetic energy by individual discrete portions (quanta) [3]. N. Bohr (Fig. 2) at the same time formulated a new physical idea that the atom of substance may be present in a number of discrete stationary energy states are not accompanied by the emission (absorption) of electromagnetic energy by it $[3,4,7]$.

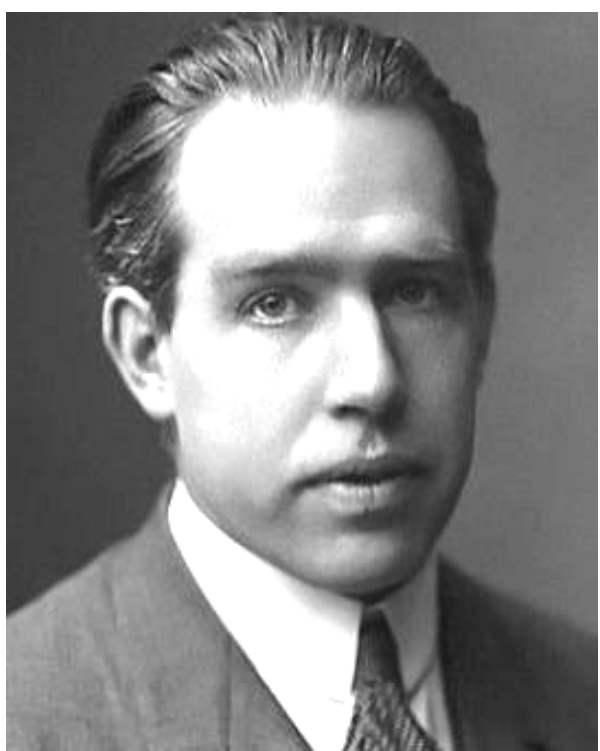

Fig. 2. Prominent Danish theoretical physicist Niels Bohr (1885-1962)

Furthermore, according to the hypothesis put forward by N. Bohr atomic transition of a substance from the normal (stationary) to an excited energy state can only occur due to the absorption of its bound electrons, rotating in the area of nuclear power of the skin in circular orbits around the nucleus of an atom, the quantum of energy (light) strictly defined value $[2,4]$. In the case of bound electron absorption of the quantum of energy he had to move to a more distant from the nucleus of an atom circular orbit. Radiating in this orbit thus obtained quantized electromagnetic energy before they $\Delta E_{n}$ the electron is forced to make an abrupt transition from the core to the remote orbit on its original and located closer to the nucleus orbit radius $r_{n}$, where $n=1,2,3, \ldots$ - integer quantum number equal to the sequence number of the electron orbit in an atom as the distance it from its core $[4,7]$. We should not confuse this with the number $n$ a principal quantum number, corresponding to the number of electron shells in the atom number and the period of the atom in the periodic table of elements by D.I. Mendeleev. Moreover, this transition is bound electron, emitting a quantum of energy with frequency $v_{n}=\Delta E_{n} / h$ it had to do without going through all the intermediate and discrete set of the proposed model of circular electron orbits of the atom. That this was the basic idea of developed by N. Bohr quantum-nuclear model of the atom. In $1922 \mathrm{~N}$. Bohr for the creation of the model of the atom, which had fundamental importance for the description of subatomic processes, was awarded [3, 5] the Nobel Prize in physics. This model of the atom was successfully used in the description of subatomic processes for the simplest atom of matter - hydrogen isotope protium ${ }_{1}^{1} \mathrm{H}$ around which a nucleus consisting of one proton $p^{+}$, single electron $e^{-}$moves in a circular orbit. Quantum theory of the Bohr atom fully satisfy the fundamental law of spectroscopy obtained experimentally by physicists and use the Rydberg constant is numerically 
equal to $R=3.289842 \cdot 10^{15} \mathrm{~s}^{-1}$ [4]. It allows with high accuracy to calculate the above-mentioned constant $R=m_{e} e_{0}^{4}\left(8 \varepsilon_{0}{ }^{2} h^{2}\right)^{-1}$ based on the four fundamental values: the electron charge module $e_{0}=1.602 \cdot 10^{-19} \mathrm{~K}$, the rest mass of the electron $m_{e}=9.109 \cdot 10^{-31} \mathrm{~kg}$, the electric constant $\varepsilon_{0}=8.854 \cdot 10^{-12} \mathrm{~F} / \mathrm{m}$ and the Planck constant $h=6.626 \cdot 10^{-34} \mathrm{~J} \cdot \mathrm{s}$. This quantum theory of the atom of substance to ensure the correct physical and quantitative interpretation of experimentally observed previously in the laboratory physicists line emission spectra for hydrogen isotope ${ }_{1}^{1} H$ (protium), including Lyman, Balmer, Paschen, Brackett and Pfund series [4, 7].

Besides, it should be noted, however, that the quantum theory of the atom by Bohr received in 1914 and its direct experimental confirmation in the famous experiments of German experimental physicists James Franck (1882-1964) and Gustav Hertz (1887-1975) for the detection of discrete excited states of a number of atoms (e.g., mercury $\mathrm{Hg}$ ) and determining their ionization energy $[2,4,6]$. Experimental results by J. Franck and G. Hertz significantly indicated that the energy of the atoms changes discretely quantized way with a strictly specific to each type of atom energy portions. So, for mercury atoms $\mathrm{Hg}$, this portion of (quantum) of electromagnetic energy, causing their excitation and subsequent emission of a photon of energy out of the transition in the ground state, was about $4.9 \mathrm{eV}$ which corresponds to a photon energy of ultraviolet light with a wavelength of $253.6 \mathrm{~nm}$ $[4,6]$. For outstanding results of years of nuclear research J. Franck and G. Hertz were awarded the Nobel Prize in physics for $1925[3,6]$.

3. Albert Einstein and the creation of the quantum theory of the photoelectric effect. As is known, the phenomenon of the photoelectric effect is the change in the electrical properties of a substance by electromagnetic radiation (light, gamma rays, ultraviolet, $\mathrm{X}$-ray, and other rays) [8]. In physics today known [4, 7]: the external photoelectric effect (the emission of the substance outside the electrons when exposed to light); internal photoelectric effect (change in electrical resistance of matter under the influence of the quanta of the electromagnetic field); photovoltaic effect (the appearance of the electromotive force in the matter under the influence of solar radiation); nuclear photoelectric effect (the emergence of a nuclear reaction in the material under the action of gamma rays). Next, we will focus on the external photoelectric effect or photoelectric effect. It is known that this effect was observed in 1887, one of the founders of classical electrodynamics, the eminent German physicist Heinrich Hertz (1857-1894) the first experimentally proved in 1888 the existence of electromagnetic waves $[3,6]$. Thus Hertz found that electric discharge in the air between two metal electrodes occurs more rapidly or at a lower voltage between the electrodes in the case of illumination luminous flux of the discharge gap containing ultraviolet rays. The physical explanation of the photoelectric effect from the standpoint of the emerging quantum mechanics was given in 1906 by the outstanding German theoretical physicist Albert Einstein (1879-1955) shown in Fig. 3.

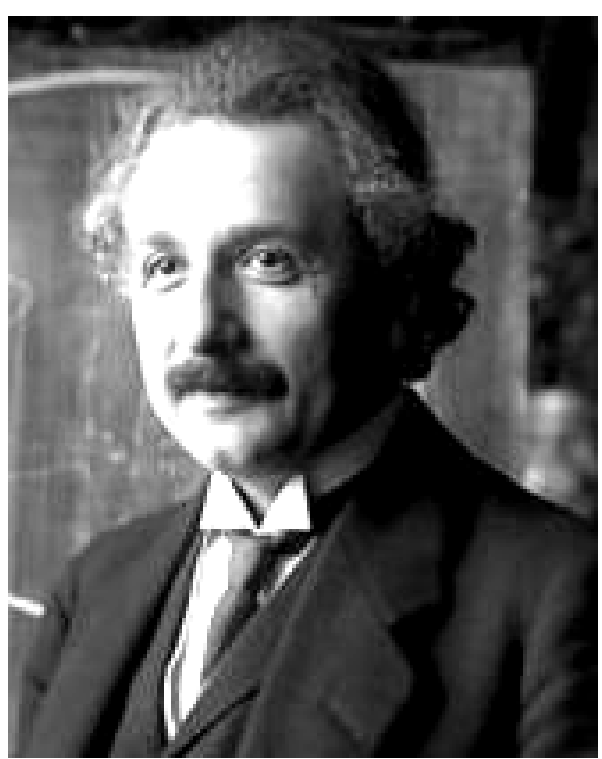

Fig. 3. Prominent German theoretical physicist Albert Einstein (1879-1955)

Improving the quantum theory by $\mathrm{M}$. Planck, A. Einstein for light emission in 1905 introduced the concept of light as a stream of quanta of the electromagnetic field or the photon flux and developed the photon theory of light [3, 4, 9]. In this theory A. Einstein based on quantum theory of electromagnetic radiation, it showed that not only the emission of light, but its distribution and absorption is performed discretely (in portions) using «light quanta». It is a new quantum-mechanical approach allowed him to formulate the law of photoemission (in physics it came as «Einstein law of the photoelectric effect») [3, 9]. For the discovering on the basis of the quantum theory of the law of the photoelectric effect, A. Einstein was awarded the Nobel Prize in physics for 1921 $[3,9]$. The experiments of the American physicist Robert Millikan performed with high accuracy in 1916 consistent with the photoelectric effect Einstein's theory [9]. A convincing proof of the discreteness of the electromagnetic energy and the existence of the photon were the results of experimental studies of another American physicist Arthur Compton (1982-1962) to change the wavelength of X-rays due to its solid-state electron scattering (Compton effect) [4]. In 1927, A. Compton for his discovery and development of the theory of «Compton quantum phenomena» was awarded the Nobel Prize in physics [3, 7].

4. Louis de Broglie and the discovery of the electron waves. In the history of physics it turned out that Louis de Broglie (Fig. 4) introduced the first scientific idea on the wave properties of the material of the microparticles in modern physics [10]. The starting point in the famous works of outstanding French theoretical physicist Louis de Broglie (1892-1987) dedicated to the 
wave properties of material microparticles became brilliant physical idea of the eminent German theoretical physicist Albert Einstein formulated it in 1905-1917 on the quantum structure of light (the «light quanta») [3, 11]. He put forward and substantiated the hypothesis of dualism of universality in microcosm $[10,11]$.

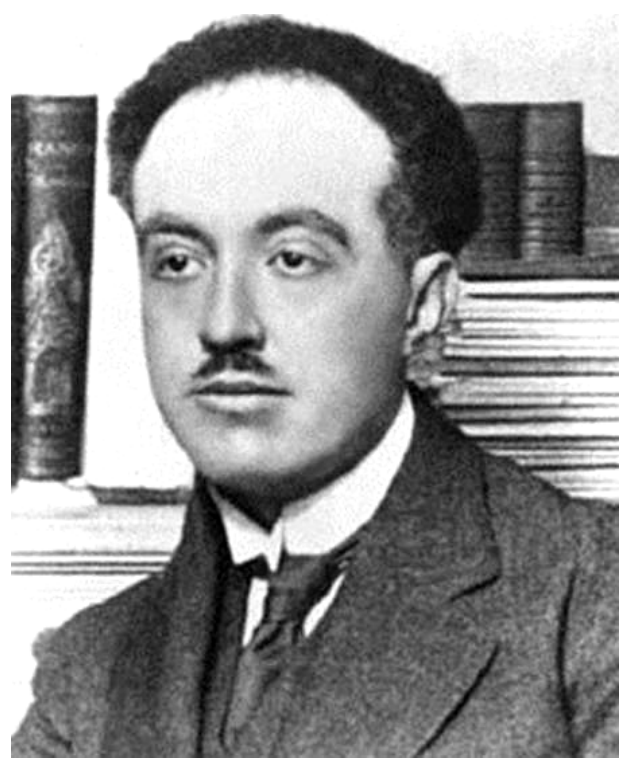

Fig. 4. Prominent French theoretical physicist Louis de Broglie (1892-1987)

In 1923 Louis de Broglie in his three research papers carried a significant development of the idea of Einstein about the dual nature of light. His original approach, he connected with the transfer of wave-particle duality of light (its simultaneous duality of particles and waves) on the microparticle substances with a rest mass. Historically, it turned out that the idea of the wave nature of matter belongs to the talented scientist Einstein once wrote [10]: «... De Broglie was the first to realize the close physical and formal relationship between quantum states of matter and resonance phenomena in the days when the wave nature of matter was not discovered experimentally». Each particle of matter has to comply with a wave of matter. This bold and revolutionary idea of Louis de Broglie has allowed him to gain which has now become a classic relationship for $\lambda_{e}$ wavelength nonrelativistic electrons in the form $[4,7]: \lambda_{e}=h /\left(m_{e} v_{e}\right)$, where $v_{e}$ is the electron velocity. Young Louis de Broglie supposes that [10]: «... electron can no longer be regarded as a mere speck of electricity. I wave to be associated with it «. These physical results Louis de Broglie summarized in 1924 in his doctoral thesis on «Studies on the quantum theory» which he successfully defended the University of Paris (the famous «Sorbonne») $[3,6]$. For the discovery of the wave nature of the electron de Broglie was awarded the Nobel Prize in physics for 1929 [3]. During the ceremony of presentation and awarding him the prize they said [10]: «... De Broglie discovered a completely new aspect of the nature of matter, which previously no one suspected. Brilliant guess de Broglie allowed long-running dispute, finding that there are not two worlds, one - light and waves, the other - matter and corpuscles. There is only one common world». What a wise and deep philosophical and physical thought!

Experimental confirmation of the hypothesis of de Broglie wave properties of electrons was carried out outstanding Britain experimental physicist George Thomson (1892-1975) - the son of the previously mentioned master of the world of physics Joseph John Thomson, who, irrespective of American experimental physicists Clinton Davisson (1881-1958) and Lester Germer (1896-1971) in 1927 discovered the diffraction of low-energy electrons («cathode rays») on a single crystal of nickel $N i[3,4]$. During his experiments, J. Thompson revealed that he had received the diffraction pattern is very reminiscent of the already known by the time the diffraction of «X-rays». For this remarkable discovery J. Thomson and K. Davisson were awarded the Nobel Prize in physics for $1937[3,6]$.

5. Erwin Schrödinger and the creation of wave mechanics. Based on the laws of classical theoretical mechanics, the Austrian theoretical physicist Erwin Schrödinger (Fig. 5) in 1926 published his famous four papers which set out a new approach to solving problems of quantum physics for nuclear facilities $[3,6]$. In them, he challenges to quantum theory of the microworld reduced to the problem of mathematical physics at the eigenvalues of some function, which he called «wave function» $[6,12]$.

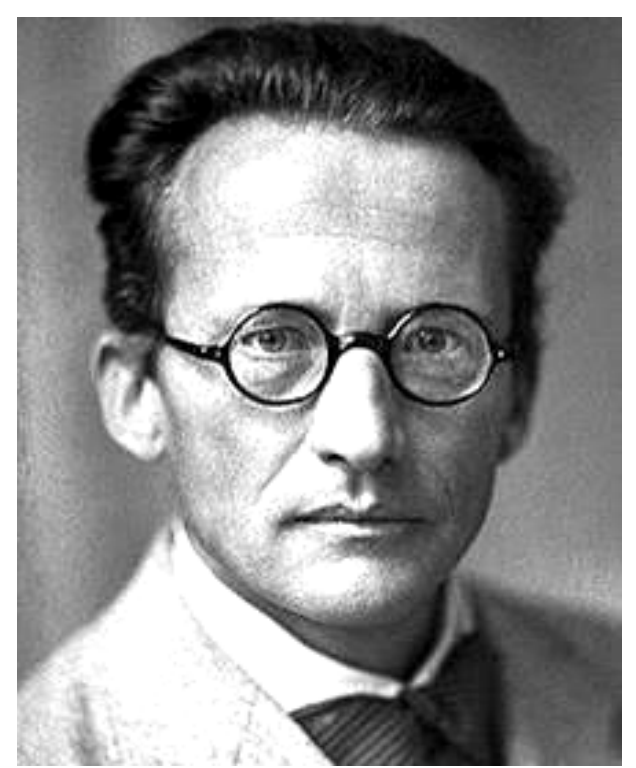

Fig. 5. Prominent Austrian theoretical physicist Erwin Schrödinger (1887-1961)

For the mathematical obtaining of the wave function ( $\psi$-function) with respect to the atom of substance, he received the corresponding partial differential equation of the second order (in the history of physics is entered as «Schrödinger equation» [3,6]), which describes the behavior of bound electrons in the well-known to him 
planetary-nuclear model of the atom $[4,12,13]$. This is the Schrödinger wave equation turned out to be a generalization and application of these earlier «wave ideas» the French theoretical physicist Louis de Broglie to the hydrogen-like atom [4]. The solution of this equation is reduced to a standing electron waves in a specified atom $[4,6]$.

Creating the wave (quantum) mechanics allowed E. Schrödinger a fresh look at the atomic and subatomic processes theory. Applying the Schrödinger wave equation to describe the quantum mechanical processes in hydrogen atoms of the material showed that the distribution of bound electrons in atomic spherical shell is subject to the respective quantized wave $\psi_{n}$-function describing in three-dimensional space of a standing wave of matter (de Broglie wave). Moreover, in this case, each quantized wave $\psi_{n}$-function matched strictly certain quantized energy of a bound electron, indicating its energy level. With such a quantum-physical approach to the analysis of intra-process it turned out that a bound electron atom considered able to move from one energy state, which corresponds to a quantized wave $\psi_{n}$ - function into another energy state described by another quantized wave $\psi_{n}$-function $[4,6]$. Moreover, received thus on the basis of exact solutions of the Schrödinger wave equation for the hydrogen isotope ${ }_{1}^{1} H$ (protium) the eigenvalues of the energy of its associated $s$-electron $\left(E_{s}=-13.6 \mathrm{eV}\right)$ and the most probable radius of its orbit $\left(r_{s}=0,529 \cdot 10^{-10} \mathrm{~m}\right)$ fully coincided with calculated results arising from quantum-nuclear model of the atom by Bohr [4, 7]. In fact, by E. Schrödinger new quantum mechanical model of the atom (in the history of physics it came as Schrödinger quantum-wave model of the atom) has been developed on the basis of the laws of wave mechanics [3, 4, 13]. For the development of new forms of atomic theory (wave mechanics) Schrödinger was honored for 1933 the Nobel Prize in physics [3]. According to this model, the atom distribution of electrons in its atomic shell described quantized wave $\psi_{n}$-functions are spatially standing waves [4]. From atomic model by Schrödinger it followed that associated electron in the atomic shell in a stationary orbit can not radiate energy on the grounds that his condition is determined by said electronic standing waves. In theory, Bohr postulated such a position only with no physical explanation. [4] The new atomic theory Schrödinger explained the phenomenon of displacement of the energy levels of the atom under the influence of strong external electric field (Stark effect, opened in 1913 [2, 4, 7]). Quantum theory of Schrödinger correctly explained the spectral lines in the hydrogen atom [4]. This quantum theory was a major scientific discovery of the first half of the 20th century in the field of nuclear physics processes.

American physicists Clinton Davisson and Lester Germer examining at the end of 1927 the scattering of electrons on a single crystal of nickel $\mathrm{Ni}$ and comparing them received experimental data with the calculated results of the well-known formula in materials by BraggWulf experimentally confirmed the existence of electron waves in the nature of matter. In addition, some of the first experimental evidence of quantum (wave) mechanics also were physical experiences of the German scientist Otto Stern carried out in 1929. These experiments have been associated with the study of the wave nature of neutral atoms and molecules, scattered on the twodimensional diffraction grating $L i F$ crystal $[4,6]$. O. Stern when it was shown that light atoms ${ }_{1}^{1} H$ isotope of hydrogen (protium) and helium ${ }_{2}^{4} \mathrm{He}$ under these conditions, there is a clear diffraction pattern, and for the heavy atoms of matter having a small «de Broglie» wavelength diffraction picture is fuzzy (vague). Moreover, in the experiments conducted by the physical intensity of the peaks above us on the diffraction patterns observed in places where the waves of matter («de Broglie» wave) going to (interfere) in the same phase [4].

6. Wolfgang Pauli and the formulation of the «exclusion principle». Austrian theoretical physicist Wolfgang Pauli (Fig. 6) has made a significant contribution to the development of quantum mechanics as the theory of physical processes and phenomena in the microcosm of nature. In the period 1924-1925 he formulated his famous «exclusion principle» (in the history of physics is the concept became a «Pauli exclusion principle» [3]). In the words of Wolfgang Pauli [14]: "... in the atom can be two electrons in which all four quantum numbers would be the same». This is the essence of this principle. Perhaps the introduction of the physical concept was a major achievement Pauli quantum theory of subatomic processes $[3,14]$.

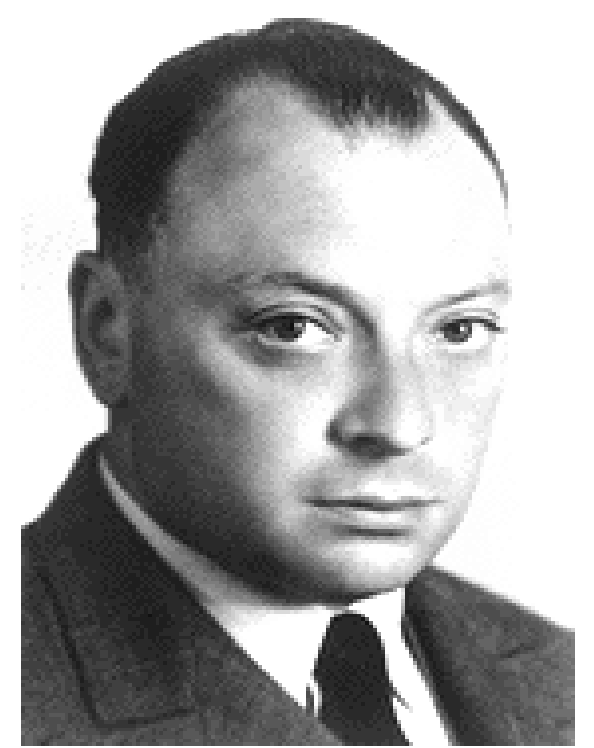

Fig. 6. Prominent Austrian theoretical physicist Wolfgang Pauli (1900-1958)

In accordance with the fundamental «Pauli exclusion principle» in the atom of substance in its electronic shells can be only one so-called bound electron, characterized by the appropriate only for him to certain 
quantitative set of four quantum numbers used in atomic physics [4, 7]: the principal quantum number $n$; orbital quantum number $l$; magnetic quantum number $m_{l}$; spin quantum number $m_{s}$. It is known that in the atom of matter quantum number $n$ determines its kinetic and potential energy, the quantum number $l$ - the shape of the electron orbit, quantum number $m_{l}$ - the position of the electron orbit in atomic space and the quantum number $m_{s}$ - its direction of circular rotation around its own axis $[4,7$ ]. This physical principle has played a huge role in determining the order of development bound electrons of the atomic shell. For scientific discovery of the «Pauli exclusion principle» outstanding theoretical physicist of the 20th century W. Pauli was awarded the Nobel Prize in physics for $1945[3,5,6]$.

7. Werner Heisenberg and the formulation of the «uncertainty relations». Prominent German physicist Werner Heisenberg (Fig. 7) is the author of a number of fundamental scientific results in quantum mechanics. In 1925 he developed a «matrix mechanics» which was the one of the theoretical aspects of quantum physics (later this mechanism was perfected by German theoretical physicists Max Born and Pascual Jordan) [3]. The starting «point» when creating this Heisenberg mechanics equivalent to E. Schrödinger's wave mechanics and the awarded of the 1932 Nobel Prize in physics [3], it was his fundamental rejection of the classical and the researcher observed subatomic processes concepts of «position» and «impulse «of the electron in an atom of matter and applying instead the concepts of «frequency «and «amplitude «of its oscillations, which the researcher can accurately be determined from optical experiment [15]. In quantum mechanics, momentum of microparticles $p=m v$, where $m$ and $v$ are respectively, the rest mass and the speed of the microparticles, with its wavelength $\lambda$ associated by de Broglie relation $(p=h / \lambda)$ [4]. As is known, the wavelength $\lambda$ is a function of the waveform and not the spatial coordinates (e.g., $z$ ). Therefore the impulse of the microparticles $p$ will not be a function of the coordinate $z$. In this regard, in quantum mechanics it is impossible to simultaneously determine the $z$ coordinate and impulse $p$ of the microparticles. In $1927 \mathrm{~W}$. Heisenberg to resolve this paradox formulated the fundamental physical principles of quantum mechanics the «uncertainty relation» named later by his name and having, for example, to conjugate variables «coordinatemomentum» form [4]: $\Delta z \cdot \Delta p \geq h /(4 \pi)$, where $\Delta z, \Delta p$ are the uncertainties in finding the position and momentum of the microparticle.

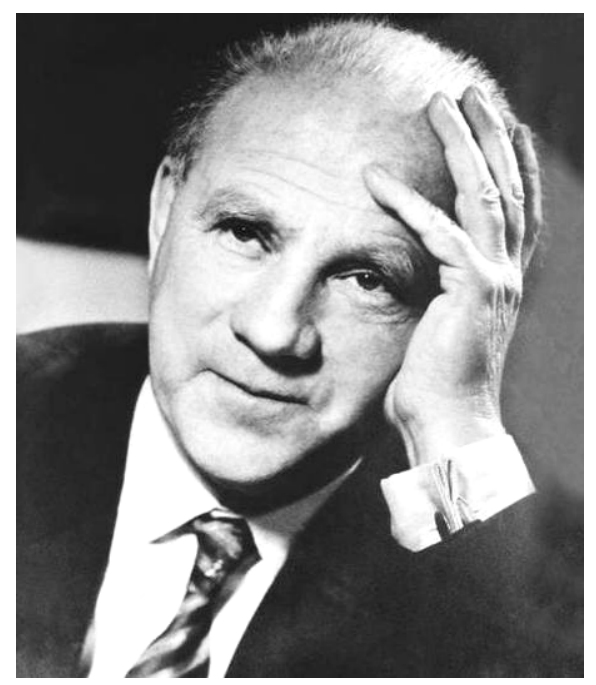

Fig. 7. Prominent German theoretical physicist Werner Heisenberg (1901-1976)

8. «Bohr's» formulation of the «principles of the relevant and complementarity». After developing his famous quantum theory of the atom, N. Bohr continued to develop this theory by trying to generalize it to the case of more complex than hydrogen ${ }_{1}^{1} H$ (protium) the manyelectron atoms. In 1918 in his paper «On the Quantum Theory of Line Spectra» N. Bohr quantitatively formulated the so-called «principle of correspondence» linking the quantum theory with classical physics $[3,16]$. He also used his idea from 1913 that in the nuclear related shell transitions of electrons between stationary orbits with large quantum numbers should provide radiation at a frequency coinciding with the frequency of revolution of the electron in the atom [16]. In formulating this principle, N. Bohr noted [16]: «... Each transition between two stationary states associated with a respective harmonic component so that the probability of the transition depends on the amplitude of oscillation. The polarization of the radiation is due to the more detailed properties of fluctuations as well as the intensity and polarization of light in the system of waves emitted by an atom from the classical theory due to the presence of said component fluctuations, determined by the amplitude and other properties of the latter»y. This principle has allowed $\mathrm{N}$. Bohr to determine the probability of transitions of electrons and hence the intensity of the spectral lines, as well as obtain the selection rule (in particular, for the harmonic oscillator) and give an interpretation of the number and the polarization component of the «Stark and Zeeman splitting» of spectral lines in strong electric and magnetic fields. The "correspondence principle» has played a huge role in the construction of a consistent quantum mechanics [16]. According to this principle, the equations of quantum physics for large quantum numbers, or with the involvement in the monitoring process of a large number of photons must coincide with the equations of classical physics to the corresponding averaged physical quantities [4]. Another important for the understanding of the physical principles and interpretation 
of the results of quantum mechanics was «complementarity principle» proposed by N. Bohr in 1927 [3, 16]. This principle reflects the logical relationship between the two methods of description or set of views for the same events in the microcosm. Indeed, on the one hand, the predictions of quantum mechanics are probabilistic. On the other hand, are used for their interpretation and presentation of the terminology of classical physics. The basis of the interpretation of quantum theory, Bohr put the wave-particle duality of microparticles which first turned his keen and insightful account of Louis de Broglie. The essence of «subsidiarity principle» is that the microparticles can not be a physical situation in which the two complementary aspects to it (for a single phenomenon in microcosm) have emerged at the same time and equally clearly [16]. In other words, in the microcosm there are no physical conditions in which micro-object would have the exact same time dynamic characteristics (for example, coordinate, momentum, energy and other values) belonging to two different specific concepts mutually exclusive. It is the scientific position and found its concrete expression in the formulated by W. Heisenberg in 1927 regardless of $\mathrm{N}$. Bohr's «uncertainty relation».

9. The creation of quantum statistics of microparticles. We begin here with the fact that we point out that in physics at the quantum statistics of mean statistical method for studying systems consisting of a large number of microparticles and obey the laws of quantum (wave) mechanics [7]. Development of this new for the first half of the 20th century physics as quantum mechanics (physics) statistical approach to describe the behavior of systems with identical (identical) microparticles faced serious scientific difficulties when trying to physics scholars of determining the number of energy states in an arbitrary gas (system microparticles) containing such microparticles that can be characterized by their «degeneracy». By this for a better understanding of the reader of the composite material should be added that the gas applied to microparticles (e.g., the «electronic gas» or so-called «electronic cloud» of the metal conductor) is considered «degenerate» in the case where the properties described by quantum-physical laws, differ significantly from the usual properties of the gas, obeying the laws of classical physics, and in accordance with laws based on its statistical physics [7]. An important step in overcoming the problems of scientific difficulties in creating quantum gas statistics, consisting of «degenerate» microparticles was made by talented theoretical physicists from Asia and Europe in the first quarter of the 20th century [3].

The quantum statistics of Bose-Einstein. The famous Indian theoretica; physicist Shatendranat Bose (1894-1974) in 1924 regardless of the outstanding German theoretical physicist Albert Einstein (1879-1955) developed the quantum statistics of identical microparticles with a zero and spin (the concept of «spin» means own mechanical angular momentum of rotating around own axis of the microparticles) received in the physics of elementary particles the name «bosons» (these include photons, phonons, and some nuclei of atoms) [3, $4,7]$. This statistics by names of its founders in quantum physics called «Bose-Einstein statistics» $[7,16]$. We point out that an important feature of bosons named after physicist S. Bose (Fig. 8) is their fundamental insubordination «Pauli exclusion principle». Therefore for bosons there are no restrictions on the number of microparticles that may be present in certain quantized energy state. With regard to the quantum statistics of the microparticles in analytical form have been received $f_{B}$ distribution functions of bosons (Bose gases) for energy, which in quantum physics became known as the BoseEinstein distribution functions $[4,7,17]$.

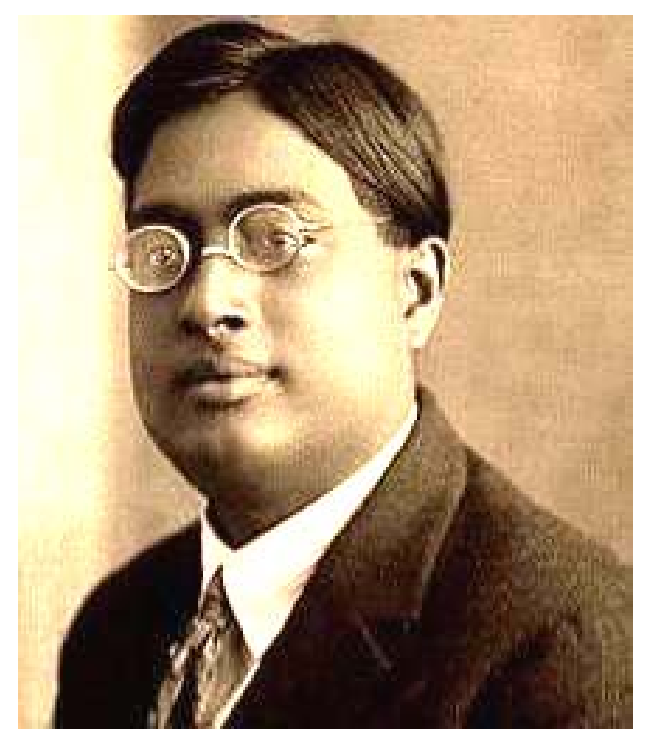

Fig. 8. Outstanding Indian theoretical physicist Shatendranat Bose (1894-1974)

Quantum Fermi-Dirac statistics. Young and in future outstanding Italian physicist Enrico Fermi (19011954) awarded in 1938 the Nobel Prize in physics for their discovery of artificial radioactivity, chemical elements, caused by the bombing of them by «slow» neutrons, in 1925 regardless of when also the young and also in future the outstanding British theoretical physicist Paul Dirac in the future (1902-1984) which became in 1933 for the discovery of new productive forms of atomic theory (for the creation of quantum mechanics) the Nobel Prize in physics [3] developed a quantum statistics for microparticles with half-integer spin (such as electrons, protons, neutrons, and other representatives of the microcosm of matter) [7]. An important feature of these microparticles (in elementary particle physics, such microparticles are now called «fermions» [4]) is that they obey the «Pauli exclusion principle» in certain quantized energy state of which can be either only one microparticle, or none microparticles. This statistic is named after talented scientists and its developers E. Fermi 
(Fig. 9) and P. Dirac (Fig. 10) in quantum physics called «Fermi-Dirac statistics» [7].

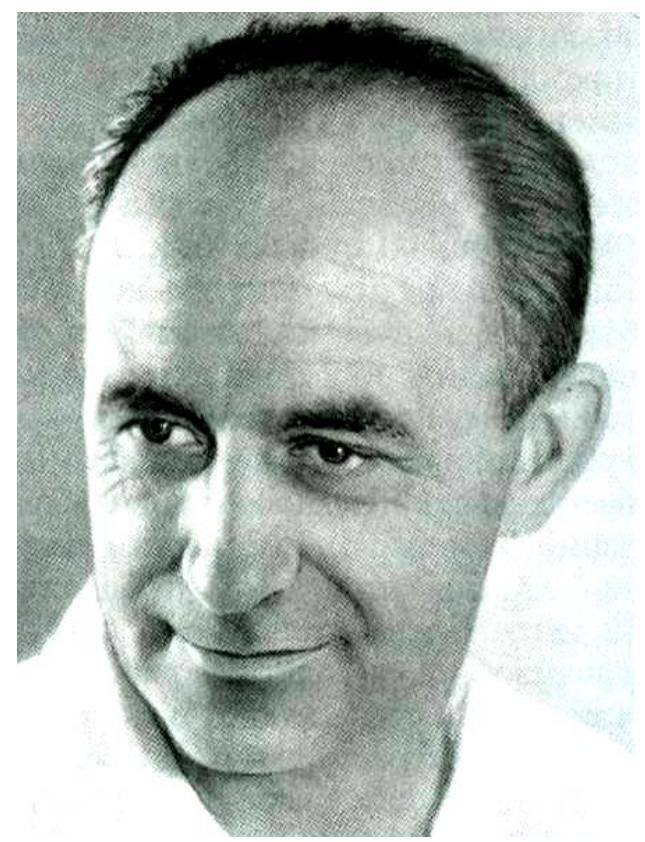

Fig. 9. Outstanding Italian theoretical physicist Enrico Fermi (1901-1954)

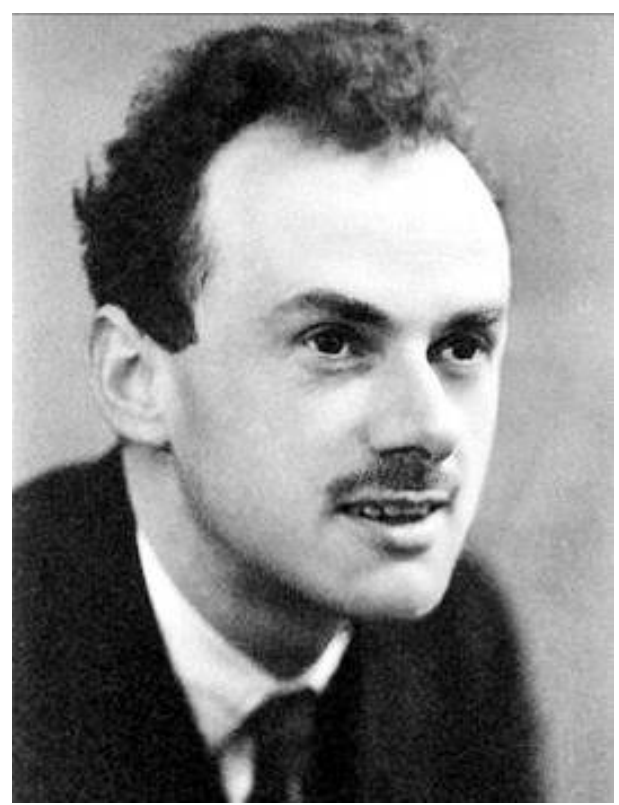

Fig. 10. Outstanding British theoretical physicist Paul Dirac (1902-1984)

Co-authors of the quantum statistics in an analytical form were obtained distribution function $f_{F}$ of fermions (Fermi gases) at energies corresponding to the average number of microparticles in the same energy state $[4,7]$. These quantum mechanical functions $f_{F}$ in quantum physics have been called the Fermi-Dirac distribution functions [7]. From a comparison of mentioned analytic functions distribution $f_{B}$ and $f_{F}$ with distribution function $f_{M B}$ by Maxwell-Boltzmann [4] characteristic of the «old» classical physics (respectively, and classical statistics) and reflecting the average number of «non-degenerate» microparticles in the same energy state, it follows that no bosons or fermions do not obey the classical distribution of microparticles in energy (velocity) in ordinary gases described previously received in the 19th century the classical distribution function $f_{M B}$ by Maxwell-Boltzmann [7]. Taking into account that fermions are part of all, without exception, we know atoms and molecules created in the first quarter of the 20th century «Fermi-Dirac statistics» becomes essential scientific and practical value for such rapidly developing worldwide knowledge areas as nuclear physics, elementary particle physics, highenergy plasma and condensed state of any matter [3, 4, 7].

10. Max Born and the formulation of the statistical interpretation of the wave function of the microparticle. In 1926, the eminent German theoretical physicist Max Born (1882-1970) Using physical idea of Einstein that light waves characterizes the square of the amplitude probability density $\rho_{F}$ of appearance of photons («quanta of the electromagnetic field») [4] He offered to the scientific world the statistical interpretation of the wave $\psi$-function by Schrödinger [3, 18]. At the same time M. Born (Fig. 11) postulated that the value of the squared modulus of the quantized wave function $\left|\psi_{n}\right|^{2}$ must be a probability density $\rho_{n}$ for stay of the microparticle (e.g., electron, proton, neutron, or other particles) in a given volume substance. Expanding physical meaning of the «Schrödinger 's» wave $\psi$-function, M. Born swung thus a kind of «bridge» between the wave and corpuscular theory of matter microparticles. Designed by M. Born physical approach was an important «step» on the way «reconciliation» of the old classic presentation «particulates of matter» with the representation of the new quantum-mechanical «waves of matter» [3]. The statistical interpretation in 1926 by M. Born of the wave $\psi$-function by Schrödinger was the first «step» on the way scientists study the probabilistic interpretation of quantum mechanics (physics), which determines the behavior of micro-particles of any substance.

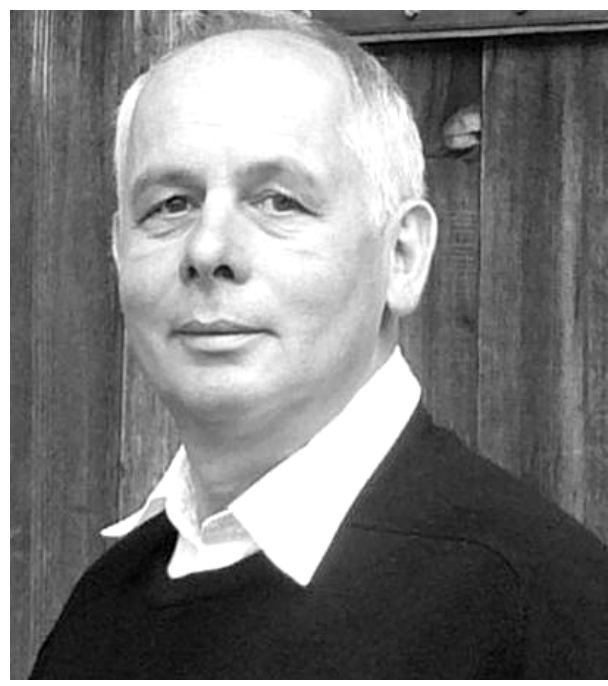

Fig. 11. Prominent German theoretical physicist Max Born (1882-1970) 
Such an interpretation of the wave $\psi$-function by Schrödinger pointed to the fundamental role of probability in the laws of the microcosm of nature around us. A more rapid recognition in the world of such an interpretation of the wave $\psi$-function by Schrödinger contributed described earlier by «Heisenberg uncertainty relation» «reconciling» the corpuscular and wave pattern of distribution of the microparticles, explaining the connection of classical mechanics to quantum mechanics and restricting the use of microscopic bodies to the concepts of classical physics. In 1954 Max Born won the Nobel Prize in physics «for outstanding results in the field of quantum mechanics» [3, 18].

11. Lev Landau and the creation of the quantum theory of the phenomenon of superfluidity of liquid helium. With the approach of the temperature of the physical body to absolute zero, and a sharp decrease in the velocity of the thermal motion of the atoms, «de Broglie» wavelength of its atoms in accordance with the formula of de Broglie and quantum uncertainty of its atomic coordinates according to the « Heisenberg uncertainty relations» become significantly more of its interatomic distances [6]. Under these conditions, a significant role in condensed matter, quantum effects begin to play and begins to manifest itself through macroscopic quantum wave nature of physical processes in the condensed state of matter («quantum fluid»). With such a mysterious manifestation of physical processes encountered physicists who received liquid helium-I with a critical temperature of $4.22 \mathrm{~K}$ and liquid helium-II of (for example, a prominent Dutch experimental physicist Heike Kamerlingh Onnes (1853-1926) who in 1911 won the Nobel Prize in physics for the discovery of superconductivity in metals $[3,6]$ ) and studying their physical properties (e.g., the prominent Soviet experimental physicist Pyotr Leonidovich Kapitsa (18941984) awarded in 1978 the Nobel Prize in physics for «fundamental inventions and discoveries in the field of low temperatures» $[3,6])$. Experiments of P.L. Kapitsa at the created by him in 1935 the Institute of Physical Problems (IPhP) of the Academy of Sciences of the USSR ended by the discovering in 1937 the phenomenon of superfluidity of liquid helium-II with a critical temperature of $2.19 \mathrm{~K}[3,4,7]$. Many attempts at domestic and foreign theoretical physicists for an explanation of the paradoxical behavior of liquid heliumII for many years remained unsuccessful. After the moving in 1937 from the Ukrainian Physico-Technical Institute (Kharkov) to work at the IPhP, Academy of Sciences of the USSR (Moscow) prominent Soviet theoretical physicist and future Academician of the Academy of Sciences of the USSR (1946) Lev Davidovich Landau (1908-1968) started close to the disclosure of the phenomenon of liquid helium-II. By 1941 L.D. Landau (Fig. 12) concludes that in liquid helium-II phase transition of II-kind not accompanied by the release (or absorption) of heat, unlike the phase transition of I-type and associated with the appearance of a substance qualitatively new specific properties $[4,19]$.

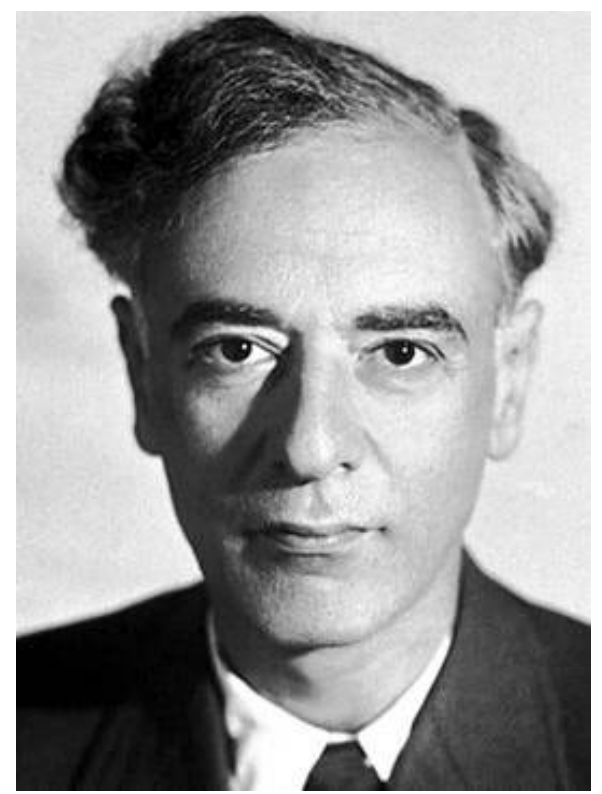

Fig. 12. Prominent Soviet theoretical physicist, Academician of the Academy of Sciences of the USSR Lev Davidovich Landau (1908-1968)

L.D. Landau in the construction of the quantum theory of liquid helium II-type of superfluidity phenomenon took advantage of the concept of «quasiparticles» («like particles») which are mainly chosen phonons (sound quanta of energy, responsible for a potential motion of matter) and rotons (quantum elementary pathogens responsible for the eddy motion of matter) $[3,6]$. In addition, L.D. Landau thus introduced the concept of the normal and superfluid component of liquid helium of II-kind. These quasi-particles is described by the behavior of the normal component in this helium condensate. This component is moved in it with friction and participated in the transfer of heat. The superfluid component moving in liquid helium II-type of friction-free and was not involved in the transfer of heat in it. These are the main characteristics of the developed by L.D. Landau in 1945 the two-fluid model of quantum liquid helium of II-kind. Completed in 1945 at the IPhP of the Academy of Sciences of the USSR low-temperature experiments involving the future Doctor of Physical and Mathematical sciences E. Andronikashvili confirmed the presence in liquid helium-II of normal and superfluid components, as well as the reliability of the whole of created by L.D. Landau the quantum theory of superfluidity of liquid helium-II [3, 6]. Taking into account the fundamental nature of this achievement, in 1962 L.D. Landau was awarded the Nobel Prize in physics «for his pioneering research in condensed matter theory, especially liquid helium» [3, 19].

12. Quantum Electronics and the creation of quantum generators of electromagnetic radiation. The term «quantum electronics» refers to a new branch of 
physics that studies the methods of amplification and generation of electromagnetic oscillations based on the use of the effect of stimulated (induced) radiation [4, 20]. Recall that in 1905 the eminent German physicist Albert Einstein based on the statistical analysis of energy fluctuations of equilibrium radiation advanced the hypothesis of «light quanta»[3]. In 1916 he came to understand the nature of equilibrium radiation in quantum systems that have discrete levels of radiation energy. At the same time by him to similar systems it introduced the concept of «stimulated emission of radiation» associated with the energy of a quantum particle transitions included in a single physical ensemble (atoms, molecules, solid, gas, etc.). In this regard, the physical basis for quantum electronics [4, 20] consists of the following three fundamental positions. The first - the energy of electromagnetic radiation consists of discrete portions of energy («light quanta» or «photons»). The second emission of light quanta in its high intensity determined by the effect of induced emission. The third - quanta of forced and forces of electromagnetic radiation (photons) are identical and are subject to quantum statistics of BoseEinstein. As a result, the quantum nature of electromagnetic radiation and the quantization of the energy levels of microparticles objectively lead to the existence of physical processes in the microscopic world, accompanied by the generation of identical each other of light quanta («bosons»). Bosonity of photons allows you to navigate in quantum electronics from the corpuscular representation of the wave, which is characterized by the principles of superposition and amplification of coherent oscillations [4, 20]. In 1927 the eminent British theoretical physicist Paul Dirac developed a consistent quantum theory of radiation (absorption) of light, the most important result of which was a rigorous justification of «stimulated emission» postulated by Albert Einstein, and its coherence [3, 21]. Despite the creation of the above-mentioned physical prerequisites for the emergence in the world of quantum electronics, until the end of 1954 in the field of quantum electronics was created at the same time in the Soviet Union (at the Laboratory of oscillations of the Physical Institute of the Academy of Sciences of the USSR under the supervision of the future Academician Alexander Mikhailovich Prokhorov (Fig. 13) and Nikolai Gennadievich Basov (Fig. 14), Moscow, Russia) and the USA (at the Laboratory of emissions of the Columbia University under the supervision of Professor Charles Townes (Fig. 15), New York city), the first «molecular quantum generator» of the microwave-induced radiation («ammonia maser») having a narrow beam of radio waves with a length of $1.27 \mathrm{~cm}$ wave $[5,21]$. In this physical device due to the use of energy physicists sorting and excitation energy ammonia molecules in the active quantum medium with population inversion, placed in the cavity, carried out a quantum jump between discrete energy levels in molecules of ammonia [4, 5]. This gas maser is very stable stimulated coherent radiation.

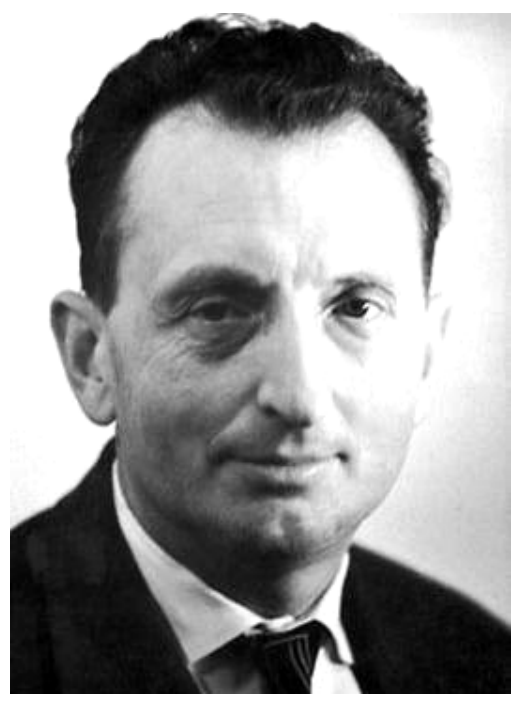

Fig. 13. Prominent Soviet experimental physicist, Academician of the Academy of Sciences of the USSR Alexander Mikhailovich Prokhorov (1916-2002)

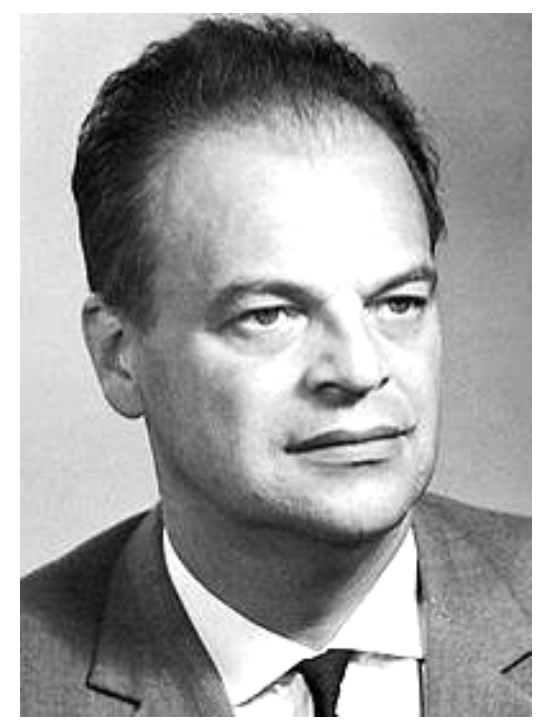

Fig. 14. Prominent Soviet experimental physicist, Academician of the Academy of Sciences of the USSR Nikolai Gennadievich Basov (1922-2001)

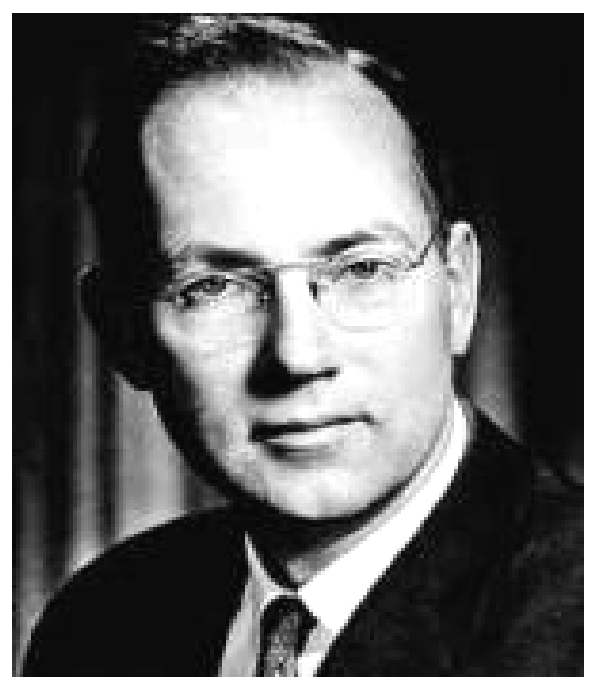

Fig. 15. Prominent American experimental physicist Charles Townes (1915-2015) 
An iimportant scientific «step» by domestic scientists-physicists in the field of quantum electronics was made in 1955 when N.G. Basov and A.M. Prokhorov proposed a new method of creating radiation in the active medium of inversion and non-equilibrium quantum systems - «three-level pump method» widely used in lasers and today [5].

Quantum electronics in the second half of the 20th century was not limited only to the development of microwave amplifiers through stimulated electromagnetic radiation («masers»). In 1960 the first solid-state «optical quantum generator» («ruby laser») was created by the American physicist T. Maiman based on the ruby crystal [5]. Then came gas, semiconductor, chemical, and other «lasers» [5, 21]. In 1964 Charles Townes, A.M. Prokhorov and N.G. Basov was awarded the Nobel Prize in physics «for fundamental work in quantum electronics, which led to the creation of masers and lasers» [3]. Currently, lasers of varying power output operate in continuous and pulsed modes, over a sufficiently wide frequency range of radiation-induced waves. Now in the world there is a huge variety of «maser» and «lasers» with various active quantum fluids and systems of excitation («pumping») [5, 20].

13. The quantum wave nature of the drift of the free electrons in metal. A convincing, clear and accessible to many electrophysicists example of the manifestation of quantum-wave properties of electrons as a microcosm of representatives are set at the macroscopic level recently in Ukraine quantum-physical features of the flow of pulsed conduction current in metals due to drift of the free electrons [22-27]. These features are manifested in the fact that, for example, in the form of aperiodic time shape $9 / 160 \mathrm{~ms}$ of relatively high density conductive structure thin galvanized wire cylindrical configuration with an axial current pulse (till $0.37 \mathrm{kA} / \mathrm{mm}^{2}$ ) for forming quantized through an electronic half-waves de Broglie wave of quantized electronic wave packages (EWP) of macroscopic dimensions there arises a periodic longitudinal thermal macrostructure consisting of alternating between a relatively «hot» and «cold» longitudinal sections with visual lockable researcher their lengths (widths) $\Delta z_{2}$. and $\Delta z_{x}$, respectively. Moreover, the width $\Delta z_{2}$ of «hot» longitudinal sections EWP of conductor correspond to said previously «Heisenberg uncertainty relations», which determines the uncertainty of the longitudinal coordinates $\Delta z$ drifting electrons with a maximum energy of approaching $W_{F}$ Fermi energy the most likely area of their location, the corresponding square module quantized wave function $\left|\psi_{e n}\right|^{2}$ for these electrons. In [22-27] it has been convincingly, both theoretically and experimentally shown to be drifting in a metal conductor with an electrical DC, AC or pulsed current of free electrons («fermions») which are quantum objects and obeying «Pauli exclusion principle», as well as meet «quantum statistics of Fermi-Dirac», because of its wave nature of the length $l_{0}$ and the radius $r_{0}$ of said circular cylindrical conductor distributed wave manner so that its length $l_{0}$ and radius $r_{0}$ always fit an integer quantized electronic half-waves of de Broglie, respectively of length $\lambda_{l n} / 2=l_{0} / n$ и $\lambda_{r n} / 2=r_{0} / n$, where $n=1,2,3, \ldots$ - integer quantum number. Quantized values $\lambda_{l n} / 2$ and $\lambda_{r n} / 2$ precisely define periodic step structure for the above quantized EWP in the conductors with the electrical current. The research results presented in [22-27], conclusively point to a clear manifestation in the metal conductors with an electric current of high density «quantum effect periodic macrolocalization free electrons», which determines the appearance of their conductive structure with EWP inhomogeneous periodic longitudinal and radial temperature fields.

Conclusion. The nature of existence and the transition of matter at the atomic and subatomic levels of its one physical state to another, chaotic (directed) motion of microparticles in the fields of physical and leakage caused by their spatial displacement of certain probabilistic micro- and macroevents determined stochastic quantum physical phenomena occurring in the microcosm any material and describes the known laws of quantum, atomic and nuclear physics, as well as the laws of physics of elementary particles, plasma, high energy and condensed state of matter. In this regard, taking into account the currently available theoretical and experimental data in the scientific world, we can confidently state that one fundamentally important scientific position that the known (yes, probably, and even unknown) physical processes and phenomena in the microcosm of matter are quantum-wave and probabilistic nature. Despite this, the quantum-wave processes which characterize the microcosm objects causally linked and determined. They can be described in the relevant differential equations uniquely determines the desired quantized wave $\psi_{n}$-functions and other necessary physical quantities in the study of the behavior and evolution we have examined microscopic representatives of nature.

\section{REFERENCES}

1. Astafurov V.I., Busev A.I. Stroenie veshchestva [Structure of matter]. Moscow, Education Publ., 1977, 160 p. (Rus).

2. Kudryavtsev P.S. Kurs istorii fiziki [The course of the history of physics]. Moscow, Education Publ., 1974, 312 p. (Rus).

3. Khramov Yu.A. Istoriia fiziki [History of Physics]. Kiev, Feniks Publ., 2006. 1176 p. (Rus).

4. Kuz'michev V.E. Zakony i formuly fiziki [Laws and formulas of physics]. Kiev, Naukova Dumka Publ., 1989. 864 p. (Rus).

5. Baranov M.I. Antologiia vydaiushchikhsia dostizhenii v nauke i tekhnike: Monografiia v 2-kh tomakh. Tom 1. [An anthology of outstanding achievements in science and technology: Monographs in 2 vols. Vol.1]. Kharkov, NTMT Publ., 2011. 311 p. (Rus).

6. Baranov M.I. Izbrannye voprosy elektrofiziki: Monografija v 2-h tomah. Tom 1: Elektrofizika $i$ vydajushhiesja fiziki mira [Selected topics electrophysics: Monographs in 2 vols. Vol.1: Electrophysics and outstanding physics of the world]. Kharkov, NTU «KhPI» Publ., 2008. 252 p. (Rus).

7. Javorskij B.M., Detlaf A.A. Spravochnik po fizike [Handbook of physics]. Moscow, Nauka Publ., 1990. 624 p. (Rus). 
8. Bol'shoj illjustrirovannyj slovar' inostrannyh slov [Large illustrated dictionary of foreign words]. Moscow, Russkie slovari Publ., 2004. 957 p. (Rus).

9. Available

https://ru.wikipedia.org/wiki/Эйнштейн,_Альберт (accessed 10 April 2014). (Rus).

10. Available at: http://100v.com.ua/en/node/2822 (accessed 12 May 2011). (Rus).

11. Available at: https://ru.wikipedia.org/wiki/Де Бройль, Луи (accessed 18 September 2013). (Rus).

12. Grashin A.F. Kvantovaja mehanika [Quantum mechanics]. Moscow, Education Publ., 1974, 207 p. (Rus).

13. Available at: https://ru.wikipedia.org/wiki/Шрёдингер, Эрвин (accessed 15 June 2012). (Rus).

14. Available

https://ru.wikipedia.org/wiki/Паули, Вольфганг (accessed 10 April 2014). (Rus).

15. Available

https://ru.wikipedia.org/wiki/Гейзенберг, Вернер (accessed 21 May 2012). (Rus).

16. Available at: https://ru.wikipedia.org/wiki/Бор, Нильс (accessed 10 May 2013). (Rus).

17. Available

https://ru.wikipedia.org/wiki/Бозе, Шатьендранат (accessed 22 February 2010). (Rus).

18. Available at: https://ru.wikipedia.org/wiki/Борн, Макс (accessed 21 April 2008). (Rus).

19. Available

at:

https://ru.wikipedia.org/wiki/Ландау, Лев Давидович

(accessed 18 September 2013). (Rus).

20. Available at: http://dic.academic.ru/dic.nsf/bse/94619 (accessed 11 May 2011). (Rus).

21. Available at: http://edu.sernam.ru/lect_qe.php?id=13 (accessed 20 August 2012). (Rus).
22. Baranov M.I. Wave distribution of free electrons in conductor with electric current of the conductivities. Electrical Engineering, 2005, no.7, pp. 25-33. (Rus).

23. Baranov M.I. New physical mechanisms and approaches in the study of the formation and distribution of the electric conduction current in the conductor. Tekhnichna elektrodynamika, 2007, no.1, pp. 13-19. (Rus).

24. Baranov M.I. Characteristic radial distribution of free electrons in a cylindrical conductor with varying electric current. Tekhnichna elektrodynamika, 2009, no.1, pp. 6-11. (Rus).

25. Baranov M.I. Features heating thin bimetallic conductor large pulse current. Elektrichestvo, 2014, no.4, pp. 34-42. (Rus).

26. Baranov M.I. Quantum-wave nature of electric current in a metallic conductor and some of its electrophysical macrophenomena. Electrical engineering \& electromechanics, 2014, no.4, pp. 25-33. (Rus). doi: 10.20998/2074-272X.2014.4.05.

27. Baranov M.I. Main characteristics of the wave distribution of free electrons in a thin metallic conductor with a pulse current of high density. Elektrichestvo, 2015, no.10, pp.20-32. (Rus).

Received 21.12.2015

M.I. Baranov, Doctor of Technical Science, Chief Researcher, Scientific-\&-Research Planning-\&-Design Institute «Molniya» National Technical University «Kharkiv Polytechnic Institute», 47, Shevchenko Str., Kharkiv, 61013, Ukraine, phone +38 057 7076841, e-mail: eft@kpi.kharkov.ua

How to cite this article:

Baranov M.I. An anthology of the distinguished achievements in science and technique. Part 34: Discovery and study of quantum-wave nature of microscopic world of matter. Electrical engineering \& electromechanics, 2016, no.5, pp. 3-15. doi: 10.20998/2074-272X.2016.5.01. 